\title{
POLYNOMIALS OF GENERATORS OF INTEGRATED SEMIGROUPS
}

\author{
RALPH DELAUBENFELS
}

(Communicated by Paul S. Muhly)

\begin{abstract}
We give general sufficient conditions on $p$ and $A$, for $p(A)$ to generate an exponentially bounded holomorphic $k$-times integrated semigroup, where $p$ is a polynomial and $A$ is a linear operator on a Banach space. Corollaries include the following.

(1) If $i A$ generates a strongly continuous group and $p$ is a polynomial of even degree with positive leading coefficient, then $-p(A)$ generates a strongly continuous holomorphic semigroup of angle $\frac{\pi}{2}$. (2) If $-A$ generates a strongly continuous holomorphic semigroup of angle $\theta$ and $p$ is an $n$th degree polynomial with positive leading coefficient, with $n\left(\frac{\pi}{2}-\theta\right)<\frac{\pi}{2}$, then $-p(A)$ generates a strongly continuous holomorphic semigroup of angle $\frac{\pi}{2}-n\left(\frac{\pi}{2}-\theta\right)$. (3) If $(-A)$ generates an exponentially bounded holomorphic $k$-times integrated semigroup of angle $\theta$, and $p$ and $\theta$ are as in (2), then $-p(A)$ generates an exponentially bounded holomorphic $(k+1)$-times integrated semigroup of angle $\frac{\pi}{2}-n\left(\frac{\pi}{2}-\theta\right)$.
\end{abstract}

\section{INTRODUCTION}

In this paper, we consider linear operators, $A$, on a Banach space, whose resolvents $(w-A)^{-1}$, are $O\left(w^{k}\right)$ outside some set $K$, and polynomials, $p$, such that $p(K)$ is contained in a sector, and show that $p(A)$ generates a holomorphic $(k+2)$-times integrated semigroup. When $p(A)$ is densely defined, it generates a $(k+1)$-times integrated semigroup (Theorem 4). This is applied to polynomials of generators of holomorphic $k$-times integrated semigroups (Theorem 9) and polynomials of generators of strongly continuous groups (Theorem 11), as described in the abstract. Even for $k=0$, that is, for polynomials of generators of strongly continuous semigroups, these results are new, except for some special cases, which we will describe below.

If $k$ is a natural number, then the strongly continuous family of bounded operators $\{S(t)\}_{t>0}$, is an exponentially bounded $k$-times integrated semigroup generated by $A$ if $S(0)=0$, and there exists real $w$ such that $(w, \infty)$ is

Received by the editors July 1, 1988 and, in revised form, December 5, 1988.

1980 Mathematics Subject Classification (1985 Revision). Primary 47D05. 
contained in the resolvent set of $A$, with

$$
(r-A)^{-1}=r^{k} \int_{0}^{\infty} e^{-r t} S(t) d t, \quad \text { for } r>w
$$

(see Arendt [1], [2], Hieber and Kellermann [8], Neubrander [10], Thieme [15]). For convenience, we will say 0-times integrated semigroup to mean a strongly continuous semigroup. When $A$ has nonempty resolvent set, $A$ generates an exponentially bounded $k$-times integrated semigroup if and only if the abstract Cauchy problem $u^{\prime}(t)=A(u(t)), u(0)=x$ has a unique exponentially bounded solution, for all $x$ in $D\left(A^{k+1}\right)$ (see Neubrander [10], or [5]).

It is well-known that the square of a generator of a strongly continuous group generates a $C_{0}$ holomorphic semigroup of angle $\frac{\pi}{2}$. (This is the special case of Theorem 11, when $p(t)=t^{2}$. See Goldstein [7, Chapter 2, 8.7].) The bounded holomorphic semigroup analogue of Theorem 9, for the special case of $p(t)=t^{n}$, is in [3], where a different method of proof was used. (See Corollary 5 of this paper.) The special case of Theorem 9, when $p(t)=t^{2}$, appears in Goldstein [6]. In that paper, it is also shown that, if $-A$ generates a cosine function, then $-A^{2 n}$ generates a $C_{0}$ holomorphic semigroup of angle $\frac{\pi}{2}$, for $n=1,2, \ldots$.

All operators are linear, on a Banach space, $X$. We will write $e^{t A}$ for the semigroup generated by $A, D(A)$ for the domain of $A$. Basic material on strongly continuous semigroups may be found in Goldstein [7], Pazy [14], or van Casteren [16]. When $p$ is a polynomial, $p(A)$ is defined in the obvious way: if $p(t)=\sum_{k=0}^{n} a_{k} t^{k}$, then $p(A) \equiv \sum_{k=0}^{n} a_{k} A^{k}$, with $D(p(A))=D\left(A^{n}\right)$. All polynomials are complex valued.

\section{MAIN RESUlts}

Definition 1. $S_{\theta}=\left\{r e^{i \phi}|r \geq 0,| \phi \mid<\theta\right\}$.

Definition 2. A $C_{0}$ (strongly continuous) semigroup is a $C_{0}$ holomorphic semigroup of angle $\theta\left(0<\theta \leq \frac{\pi}{2}\right)$ if it extends to a semigroup holomorphic in the interior of $S_{\theta}$, and continuous on $\bar{S}_{\psi}$, whenever $\psi<\theta$.

A $C_{0}$ holomorphic semigroup of angle $\theta$ is a bounded holomorphic semigroup (BHS) of angle $\theta$ if it is bounded on $S_{\psi}$, whenever $\psi<\theta$.

Remark. It is well-known (see any of the references for $C_{0}$ semigroups) that $-A$ generates a BHS of angle $\theta$ if and only if $D(A)$ is dense, the spectrum of $A$ is contained in $\bar{S}_{\pi / 2-\theta}$ and for all $\psi>\left(\frac{\pi}{2}-\theta\right),\left\{\left\|w(w-A)^{-1}\right\| \mid w \notin \bar{S}_{\psi}\right\}$ is bounded.

Definition 3. Suppose $\frac{\pi}{2} \geq \theta>0$. The $k$-times integrated semigroup $S(t)$ is an exponentially bounded holomorphic $k$-times integrated semigroup of angle $\theta$ if it extends to a family of operators $\{S(z)\}_{z \in S_{\theta}}$ satisfying

(1) The map $z \rightarrow S(z)$, from $S_{\theta}$ into $B(X)$, is holomorphic.

(2) $d^{k} / d z^{k} S(z)$ is a semigroup. 
(3) For all $\psi<\theta, S(z)$ is strongly continuous on $\bar{S}_{\psi}$.

(4) For all $\psi<\theta$, there exist finite $M_{\psi}, w_{\psi}$ such that $\|S(z)\| \leq M_{\psi} e^{w_{\psi}|z|}$, for all $z$ in $S_{\psi}$.

Remark. Essentially the same idea, as appears in Definition 3, when $D(A)$ is dense, is in Okazawa [13], where semigroups of class $\left(H_{n}\right)$ are defined.

Remark. In [4], we show that, if $D(A)$ is dense, then $-A$ generates an exponentially bounded holomorphic $k$-times integrated semigroup of angle $\theta>0$ if and only if for all $\psi>\left(\frac{\pi}{2}-\theta\right)$, there exists real $c_{\psi}$ such that the spectrum of $A$ is contained in $c_{\psi}+S_{\psi}$, with $\left\{\left\|w^{1-k}(w-A)^{-1}\right\| \mid w \notin c_{\psi}+S_{\psi}\right\}$ bounded. When $D(A)$ is not dense, this condition is sufficient to guarantee that $-A$ generates an exponentially bounded holomorphic $\cdot(k+1)$-times integrated semigroup of angle $\theta$.

Theorem 4. Suppose $K$ is a subset of the complex plane containing the spectrum of $B, k$ is a nonnegative integer, $\frac{\pi}{2}>\theta>0,\left\{\left\|w^{1-k}(w-A)^{-1}\right\| \mid w \notin K\right\}$ is bounded, and $q$ is a polynomial such that $q(K)$ is contained in $S_{\theta}$. Then

(a) $-q(A)$ generates an exponentially bounded holomorphic $(k+1)$-times integrated semigroup of angle $\left(\frac{\pi}{2}-\theta\right)$.

(b) If $D(q(A))$ is dense, then $-q(A)$ generates an exponentially bounded holomorphic $k$-times integrated semigroup of angle $\left(\frac{\pi}{2}-\theta\right)$.

(c) If $k=0, D(q(A))$ is dense, and $q(0)=0$, then $-q(A)$ generates a BHS of angle $\left(\frac{\pi}{2}-\theta\right)$.

Proof. There exists finite $M$ such that

$$
\left\|(w-A)^{-1}\right\| \leq M|w|^{k-1}, \quad \forall w \notin K .
$$

Let $p(t)=q(t)-q(0), V=S_{\theta}-q(0)$. To prove the theorem, it is sufficient to show that the spectrum of $p(A)$ is contained in $V$, with $\left\{\left\|z^{1-k}(z-p(A))^{-1}\right\| \mid z \notin V\right\}$ bounded (see Remarks after Definitions 2 and 3 ).

Suppose $z$ is not in $V$. Let $\left\{w_{j}\right\}_{j=1}^{N}$ be the (not necessarily distinct) zeroes of $z-p(w)$, that is,

$$
z-p(w)=\prod_{j=1}^{N}\left(w_{j}-w\right), \quad \forall \text { complex } w .
$$

We have

$$
z-p(A)=\prod_{j=1}^{N}\left(w_{j}-A\right) .
$$

For any $j$, since $p\left(w_{j}\right)=z$ is not in $V, w_{j}$ is not in $K$. Thus $\left(w_{j}-A\right)$ is invertible, and $\left\|\left(w_{j}-A\right)^{-1}\right\| \leq M\left|w_{j}\right|^{k-1}$. Thus, $z-p(A)$ is invertible, and 
we obtain the following upper bound for $(z-p(A))^{-1}$.

$$
\begin{aligned}
\left\|(z-p(A))^{-1}\right\| & \leq \prod_{j=1}^{N}\left\|\left(w_{j}-A\right)^{-1}\right\| \\
& \leq M^{N} \prod_{j=1}^{N}\left|w_{j}\right|^{k-1} \\
& =M^{N}|z|^{k-1} .
\end{aligned}
$$

Thus, $\left\{\left\|z^{1-k}(z-p(A))^{-1}\right\| \mid z \notin V\right\}$ is bounded, proving the theorem.

As an immediate corollary, we get the results of [3].

Corollary 5. Suppose $-A$ generates a BHS of angle $\theta$, and $n\left(\frac{\pi}{2}-\theta\right)<\frac{\pi}{2}$. Then $-A^{n}$ generates a BHS of angle $\frac{\pi}{2}-n\left(\frac{\pi}{2}-\theta\right)$.

Proof. Suppose $\frac{\pi}{2}>\psi>n\left(\frac{\pi}{2}-\theta\right)$. Then, since $-A$ generates a BHS of angle $\theta$, the spectrum of $A$ is contained in $S_{\psi / n}$, and $\left\{\left\|z(z-A)^{-1}\right\| \mid z \notin S_{\psi / n}\right\}$ is bounded.

Let $q(t)=t^{n}$. Since $A$ generates a BHS, $D(q(A))$ is dense. Also $q\left(S_{\psi / n}\right)$ is contained in $S_{\psi}$, so by Theorem $4(c),-A^{n}=-q(A)$ generates a BHS of angle $\frac{\pi}{2}-\psi$, whenever $\frac{\pi}{2}>\psi>n\left(\frac{\pi}{2}-\theta\right)$. This implies that $-A^{n}$ generates a BHS of angle $\frac{\pi}{2}-n\left(\frac{\pi}{2}-\theta\right)$.

In order to apply Theorem 4 to more general polynomials of other generators, we need some elementary lemmas.

Lemma 6. Suppose $E$ is a subset of the complex plane, and $\theta \geq 0$. Then

$$
\varlimsup_{R \rightarrow \infty} \sup \{|\arg (z)||z \in E,| z \mid=R\} \leq \theta
$$

if and only if for all $\psi>\theta$, there exists real $c_{\psi}$ such that $E$ is contained in $c_{\psi}+S_{\psi}$.

Proof. Suppose the $\overline{\lim }$ inequality holds, and $\psi>\theta$. There exists finite $M$ such that $|\arg (z)|<\psi$, when $z$ is in $E$ and $|z| \geq M$. Thus, $E$ is contained in $S_{\psi} \cup\{z \in \mathbf{C}|| z \mid \leq M\}$, which may be shown to be contained in $-M(1+\cot \psi)+S_{\psi}$.

Conversely, suppose that, for all $\psi>\theta$, there exists real $c_{\psi}$ such that $E$ is contained in $c_{\psi}+S_{\psi}$. For any $\psi \leq \pi$, it is not hard to see that $\varlimsup_{R \rightarrow \infty} \sup \left\{|\arg (z)|\left|z \in c_{\psi}+S_{\psi},\right| z \mid=R\right\}$ equals $\psi$. Thus

$$
\varlimsup_{R \rightarrow \infty} \sup \{|\arg (z)||z \in E,| z \mid=R\} \leq \psi,
$$

for all $\psi>\theta$, which concludes the proof.

Lemma 7. If $p(t)=t^{n}+q(t)$, where $q$ is a polynomial of degree less than $n$, $\theta \geq 0$ and $c$ is real, then, for all $\psi>n \theta$, there exists real $c_{\psi}$ such that $p\left(c+S_{\theta}\right)$ is contained in $c_{\psi}+S_{\psi}$. 
Proof. $\lim _{|z| \rightarrow \infty} p(c+z) / z^{n}=1$. Thus,

$$
\begin{aligned}
\varlimsup_{R \rightarrow \infty} & \sup \left\{|\arg (z)|\left|z \in p\left(c+S_{\theta}\right),\right| z \mid=R\right\} \\
= & \varlimsup_{R \rightarrow \infty} \sup \left\{|\arg (p(c+z))|\left|z \in S_{\theta},\right| z \mid=R\right\} \\
= & \varlimsup_{R \rightarrow \infty} \sup \left\{\left|\arg \left(z^{n}\right)\right|\left|z \in S_{\theta},\right| z \mid=R\right\} \\
= & n \theta .
\end{aligned}
$$

Applying Lemma 6 now gives the result.

Lemma 8. Suppose $K$ equals $-c+S_{\theta} \cup c-S_{\theta}$, where $c$ and $\theta$ are nonnegative, and $p(t)=t^{2 n}+q(t)$, where $q$ is a polynomial of degree less than $2 n$. Then, for all $\psi>2 n \theta$, there exists real $c_{\psi}$ such that $p(K)$ is contained in $c_{\psi}+S_{\psi}$.

Proof. Let $K^{+}=-c+S_{\theta}$. Since $K=K^{+} \cup-K^{+}$, it is sufficient, by Lemma 6 , to show that $\varlimsup_{r \rightarrow \infty} \sup \left\{|\arg (p(z))| \mid z \in \pm K^{+}\right\}=2 n$; this follows exactly as in the proof of Lemma 7.

Theorem 9. Suppose $-A$ generates an exponentially bounded holomorphic $k$-times integrated semigroup of angle $\theta, p(t)=t^{n}+q(t)$, where $q$ is a polynomial of degree less than $n$, and $n\left(\frac{\pi}{2}-\theta\right)<\frac{\pi}{2}$. Then

(a) $-p(A)$ generates an exponentially bounded holomorphic $(k+1)$-times integrated semigroup of angle $\frac{\pi}{2}-n\left(\frac{\pi}{2}-\theta\right)$.

(b) If $D(p(A))$ is dense, then $-p(A)$ generates an exponentially bounded holomorphic $k$-times integrated semigroup of angle $\frac{\pi}{2}-n\left(\frac{\pi}{2}-\theta\right)$.

(c) If $k=0$, then $-p(A)$ generates a $C_{0}$ holomorphic semigroup of angle $\frac{\pi}{2}-n\left(\frac{\pi}{2}-\theta\right)$.

Proof. Suppose $\frac{\pi}{2}>\psi>n\left(\frac{\pi}{2}-\theta\right)$. Choose $\phi$ such that $\frac{\psi}{n}>\phi>\frac{\pi}{2}-\theta$. Since $-A$ generates an exponentially bounded holomorphic $k$-times integrated semigroup of angle $\theta$, there exists real $c$ such that the spectrum of $A$ is contained in $c+S_{\phi}$, and $\left\{\left\|w^{1-k}(w-A)^{-1}\right\| \mid w \notin c+S_{\phi}\right\}$ is bounded.

By Lemma 7, there exists real $c_{\psi}$ such that $p\left(c+S_{\phi}\right)$ is contained in $c_{\psi}+S_{\psi}$. By Theorem $4(a), c_{\psi} I-p(A)$ generates an exponentially bounded holomorphic $(k+1)$-times integrated semigroup of angle $\frac{\pi}{2}-\psi$.

Thus, whenever $\frac{\pi}{2}>\psi>n\left(\frac{\pi}{2}-\theta\right),-p(A)$ generates an exponentially bounded holomorphic $(k+1)$-times integrated semigroup of angle $\frac{\pi}{2}-\psi$. This implies (a).

The same argument, using Theorem 4(b), implies (b).

For (c), note that, since $-A$ generates a $C_{0}$ semigroup, $D(p(A))$ is dense. Thus the argument above, with Theorem 4(c), implies (c).

Corollary 10. Suppose $p$ is a polynomial with positive leading coefficient.

(a) If $-A$ generates a $C_{0}$ holomorphic semigroup of angle $\frac{\pi}{2}$, then $-p(A)$ generates a $C_{0}$ holomorphic semigroup of angle $\frac{\pi}{2}$. 
(b) If $-A$ generates an exponentially bounded holomorphic $k$-times integrated semigroup of angle $\frac{\pi}{2}$, then $-p(A)$ generates an exponentially bounded holomorphic $(k+1)$-times integrated semigroup of angle $\frac{\pi}{2}$.

The following theorem would follow from Theorem 9 and the fact that the square of the generator of a $C_{0}$ group generates a $C_{0}$ holomorphic semigroup, if $q(t)$ contained only even powers of $t$. Theorem 11 is more general, in that $q$ may be any polynomial of degree less than $2 n$.

Theorem 11. Suppose iA generates a strongly continuous group, and $p(t)=t^{2 n}+$ $q(t)$, where $q$ is a polynomial of degree less than $2 n$. Then $-p(A)$ generates a $C_{0}$ holomorphic semigroup of angle $\cdot \frac{\pi}{2}$.

Proof. Suppose $\frac{\pi}{2}>\phi>0$. Since $i A$ generates a $C_{0}$ group, there exists positive $r$ such that the spectrum of $A$ is contained in the horizontal strip $\{z \in \mathbf{C}|| \operatorname{Im}(z) \mid<r\}$, with $\left\{\| \operatorname{Im}(z)(z-A)^{-1}|||| \operatorname{Im}(z) \mid \geq r\right\}$ bounded.

Let $c=r \cot \left(\frac{\phi}{4 n}\right), K=\left(-c+S_{\phi / 4 n}\right) \cup\left(c-S_{\phi / 4 n}\right)$.

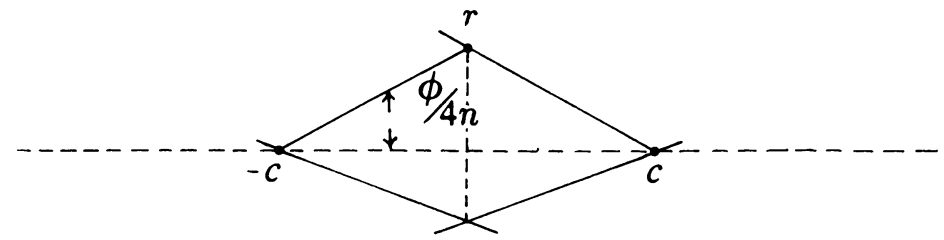

Since $\{z \in \mathbf{C}|| \operatorname{Im}(z) \mid<r\}$ is contained in $K$, and $\{\mid z / \operatorname{Im}(z) \| z \notin K\}$ is bounded, it follows that $\left\{\left\|z(z-A)^{-1}\right\| \mid z \notin K\right\}$ is bounded.

By Lemma 8, there exists real $c_{\phi}$ such that $p(K)$ is contained in $c_{\phi}+S_{\phi}$.

Since $i A$ generates a $C_{0}$ group, $D(p(A))$ is dense. By Theorem $4(\mathrm{c})$, $c_{\phi} I-p(A)$ generates a BHS of angle $\frac{\pi}{2}-\phi$. Thus, for any positive $\phi,-p(A)$ generates a $C_{0}$ holomorphic semigroup of angle $\frac{\pi}{2}-\phi$, so that $-p(A)$ generates a $C_{0}$ holomorphic semigroup of angle $\frac{\pi}{2}$.

The same argument, using Theorem 4(a) and (b), instead of (c), gives the following.

Theorem 12. Suppose both $i A$ and -iA generate exponentially bounded $k$-times integrated semigroups and $p$ is as in Theorem 11. Then

(a) $-p(A)$ generates an exponentially bounded holomorphic $(k+1)$-times integrated semigroup of angle $\frac{\pi}{2}$.

(b) If $D(p(A))$ is dense, then $-p(A)$ generates an exponentially bounded holomorphic $k$-times integrated semigroup of angle $\frac{\pi}{2}$.

Corollary 13. Suppose $p$ is an arbitrary polynomial.

(a) If both $i A$ and -iA generate exponentially bounded $k$-times integrated semigroups, then $-|p|^{2}(A)$ generates an exponentially bounded holomorphic $(k+1)$-times integrated semigroup of angle $\frac{\pi}{2}$. 
(b) If, in addition to $(a), D\left(|p|^{2}(A)\right)$ is dense, then $-|p|^{2}(A)$ generates an exponentially bounded holomorphic $k$-times integrated semigroup of angle $\frac{\pi}{2}$.

(c) If iA generates a $C_{0}$ group, then $-|p|^{2}(A)$ generates a $C_{0}$ holomorphic semigroup of angle $\frac{\pi}{2}$.

\section{EXAMPLES}

The most obvious application of Theorem 11 is to choose $A=i \frac{d}{d x}$, on $L^{p}(\mathbf{R})$, for $1 \leq p<\infty$. For $p=\infty$, the resolvent of $i A$ still satisfies the same growth conditions as the generator of a $C_{0}$ group, thus the proof of Theorem 11 with Theorem 4(a) replacing Theorem 4(c), gives us (b) of the following.

Example 1. Let $A \equiv i \frac{d}{d x}$, on $L^{p}(\mathbf{R}) \quad(1 \leq p \leq \infty)$, with maximal domain, and $B \equiv(-1)^{n}\left(\frac{d}{d x}\right)^{2 n}+q\left(i \frac{d}{d x}\right)=A^{2 n}+q(A)$, where $q$ is a polynomial of degree less than $2 n$.

(a) If $1 \leq p<\infty$, then $B$ generates a $C_{0}$ holomorphic semigroup of angle $\frac{\pi}{2}$.

(b) If $p=\infty$, then $B$ generates an exponentially bounded holomorphic once-integrated semigroup of angle $\frac{\pi}{2}$.

Remark. In Hieber and Kellermann [8], it is shown that $Q\left(i \frac{d}{d x}\right)$, on $L^{p}(\mathbf{R})$, $1 \leq p \leq \infty$, generates an exponentially bounded once-integrated semigroup, whenever $Q(\mathbf{R})$ is contained in a left half-plane.

Suppose $B$ generates an exponentially bounded $k$-times integrated semigroup. In Neubrander [11], and [5], it is shown that

$$
A \equiv\left[\begin{array}{cc}
B & B \\
0 & B
\end{array}\right], \quad D(A) \equiv D(B) \times D(B),
$$

generates an exponentially bounded $(k+1)$-times integrated semigroup. It is straightforward to show that this integrated semigroup is holomorphic if the integrated semigroup generated by $B$ is (see Neubrander and deLaubenfels [12]). Thus we have the following, using the fact that

$$
A^{n}=\left[\begin{array}{cc}
B^{n} & n B^{n} \\
0 & B^{n}
\end{array}\right]
$$

for all $n$.

Example 2. (a) Suppose both $i B$ and $-i B$ generate exponentially bounded $k$ times integrated semigroups, and $p(t)=t^{2 n}+q(t)$, where $\operatorname{deg}(q)<2 n$. Then

$$
-\left[\begin{array}{cc}
p(B) & B p^{\prime}(B) \\
0 & p(B)
\end{array}\right]
$$

generates a holomorphic exponentially bounded $(k+2)$-times integrated semigroup of angle $\frac{\pi}{2}$. If $D\left(B^{2 n}\right)$ is dense, then it generates a holomorphic exponentially bounded $(k+1)$-times integrated semigroup. 
(b) Suppose $-B$ generates an exponentially bounded holomorphic $k$-times integrated semigroup of angle $\theta$, and $p$ is an $n$th degree polynomial with positive leading coefficient, with $n\left(\frac{\pi}{2}-\theta\right)<\frac{\pi}{2}$. Then the operator in (3.2) generates an exponentially bounded holomorphic $(k+2)$-times integrated semigroup of angle $\frac{\pi}{2}-n\left(\frac{\pi}{2}-\theta\right)$. If $D\left(B^{n}\right)$ is dense, then it generates an exponentially bounded holomorphic $(k+1)$-times integrated semigroup of angle $\frac{\pi}{2}-n\left(\frac{\pi}{2}-\theta\right)$.

In Example 2(a), $B$ could be $Q\left(i \frac{d}{d x}\right)$, on $L^{p}(\mathbf{R})(1 \leq p \leq \infty)$, when $Q(\mathbf{R})$ is contained in a left half-plane (see Remark after Example 1).

\section{REFERENCES}

1. W. Arendt, Resolvent positive operators, Proc. London Math. Soc. (3) 54 (1987), 321-349.

2. __ Vector valued Laplace transforms and Cauchy problems, Israel J. Math. (3) 59 (1987), 327-352.

3. R. deLaubenfels, Powers of generators of holomorphic semigroups, Proc. Amer. Math. Soc. 99 (1987), 105-108.

4. __ Holomorphic C-semigroups and integrated semigroups, J. d'Analyse Math. (submitted).

5. __ Integrated semigroups, C-semigroups and the abstract Cauchy problem, Semigroup Forum (to appear).

6. J. A. Goldstein, Some remarks on infinitesimal generators of analytic semigroups, Proc. Amer. Math. Soc. 22 (1969), 91-93.

7. __ Semigroups of linear operators and applications, Oxford, New York, 1985.

8. M. Hieber and H. Kellermann, Integrated semigroups, J. Func. An. (to appear).

9. I. Miyadera and N. Tanaka, Some remarks on C-semigroups and integrated semigroups, Ser. A, Proc. Japan Acad. 63 (1987).

10. F. Neubrander, Integrated semigroups and their applications to the abstract Cauchy problem, Pacific J. Math. (to appear).

11. Integrated semigroups and their applications to systems of evolution equations, proceedings of a conference, in "Theory and Applications of Differential Equations," Marcel-Dekker (to appear).

12. F. Neubrander and R.deLaubenfels, Laplace transform and regularity of $C$-semigroups, preprint.

13. N. Okazawa, Operator semigroups of class $\left(D_{n}\right)$, Math. Japonicae 18 (1973), 33-51.

14. A. Pazy, Semigroups of linear operators and applications to partial differential equations, Springer, New York, 1983.

15. $\mathrm{H}$. Thieme, Integrated semigroups and duality, preprint.

16. J. A. van Casteren, Generators of strongly continuous semigroups, in "Research Notes in Math.," Pitman, 1985.

Department of Mathematics, Ohio University, Athens, Ohio 45701 